IOS Press

\title{
A study on the influence of headphones in auditory perceptual function
}

\author{
Yoshinori Horie and Takashi Toriizuka \\ College of Industrial Technology, Nihon University, \\ 1-2-1, Izumi, Narashino, Chiba, 275-8575, Japan
}

\begin{abstract}
The focus of this study is a human's ability to make full use of listening and hearing. This ability consists of dividing auditory information into a signal and a noise. To evaluate the risk of using headphones, the study investigated the auditory perception when a warning sound is given in the presence of environmental noise.
\end{abstract}

Keywords: listening, hearing, P300, headphone

\section{Introduction}

The focus of this study is a human's ability to make full use of listening and hearing. This ability consists of dividing auditory information into a signal and a noise. To evaluate the risk of using headphones, the study investigated the auditory perception when a warning sound is given in the presence of environmental noise.

\section{Method}

In an experiment, an event-related potential (P300) was measured in three conditions: 1) not using headphones, 2) using ordinary headphones, and 3) using noise-cancelling headphones. In an outdoor situation, when a subject wearing normal headphones

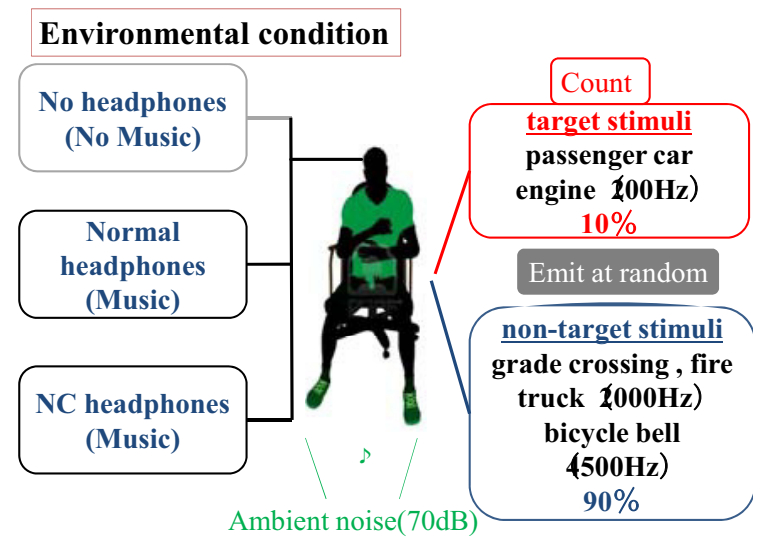

played music at a volume that prevented the hearing of ambient environmental noise (medium volume), the subject was distracted and did not pay full attention to the target stimulus sound, resulting in a great decrease in the subject's ability to distinguish the target stimulus sound from the noise.

In outdoor environments, information representing a signal may be present even in the low-frequency region of $200 \mathrm{~Hz}$. The results thus indicate that listening to music even at a low volume with $\mathrm{NC}$ headphones clearly entails risk because it precludes discrimination of the signal from noise. With NC headphones, moreover, although sounds in the $4500 \mathrm{~Hz}$ and $2000 \mathrm{~Hz}$ regions are not eliminated by the noisecancelling function, and it may therefore seem likely that discrimination of target stimuli in those frequency regions should be possible.

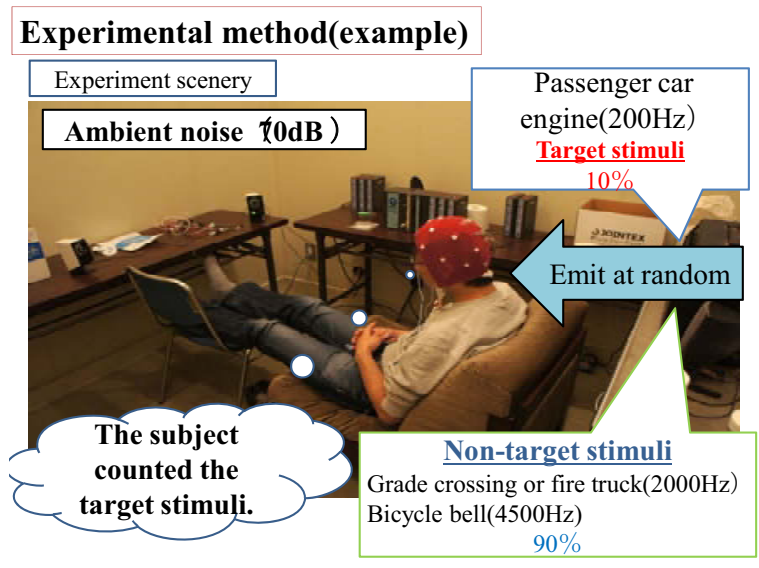




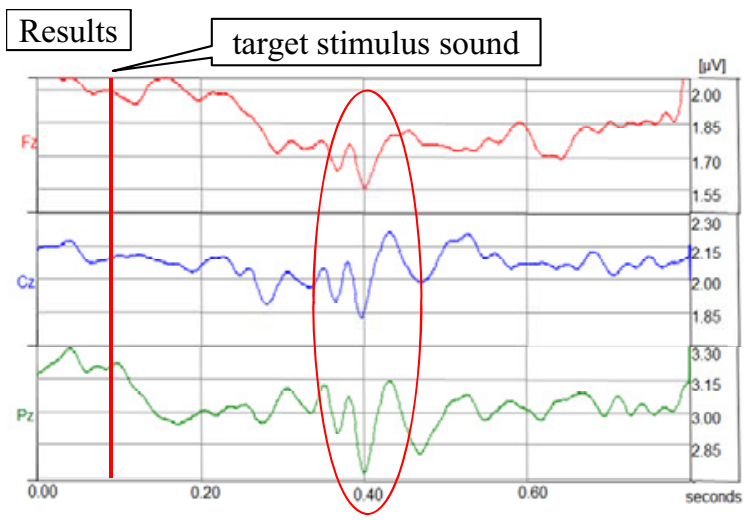

Fig 1, Headphones non-use $(\mathrm{n}=4)$ (passenger car engine $(200 \mathrm{~Hz})$ )

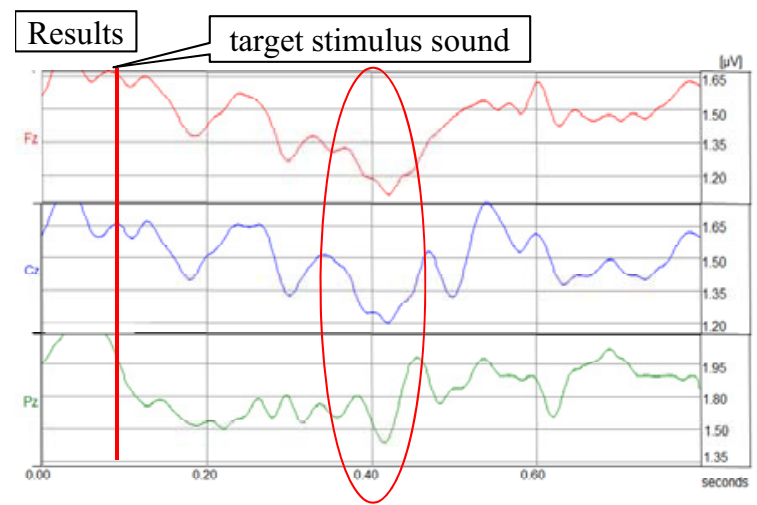

Fig 2, Normal headphones use $(n=4)$, (low (vol. 6) (Passenger car engine $(200 \mathrm{~Hz})$ )

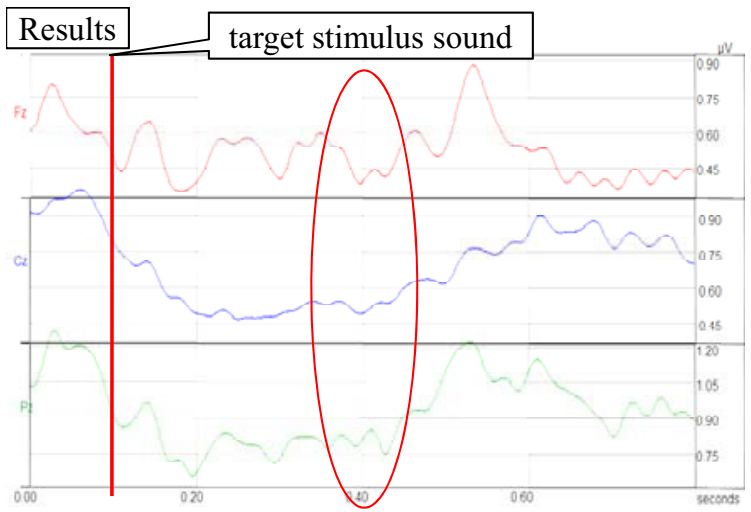

Fig 3, NC headphones use ( $\mathrm{n}=4)$ (low(vol. 6) (Passenger car engine $(200 \mathrm{~Hz}))$

\section{Results and Discussion}

The results of this study demonstrate the possibility of objectively measuring the influence of external factors on auditory perception. The results indicate that listening to music with ordinary (non-NC) headphones outdoors at a volume (vol 9) rendering ambi- ent environmental noise inaudible impedes attentiveness to target stimuli and thereby greatly reduces functional discrimination.

The results also indicate that, with $\mathrm{NC}$ headphones and listening to music at a volume (vol 6) at which ambient noise remains audible, attention to the target stimulus of a car engine $(200 \mathrm{~Hz})$ is ineffective due to active cancellation of the $200 \mathrm{~Hz}$ frequency region by the headphones. This in turn indicates that outdoor use of NC headphones entails substantial risk even with a sound volume (vol 6) at which ambient environmental sounds remain audible.

\section{Conclusion}

The results of the present study indicate that such discrimination is impeded if the music volume is increased, and so the outdoor use of NC earphones also entails substantial risk.

Further investigation is needed to determine the sound volume boundaries for target stimulus discrimination while using headphones, and the influence of musical genre and target stimulus types.

Table 1 Generation of P300 $(n=4)$

\begin{tabular}{|c|c|c|c|c|}
\hline & \multicolumn{3}{|c|}{$\begin{array}{l}\text { Volume } \\
\end{array}$} \\
\hline & & Low (vol 6) & Midium (vol 9) & Loud (vol 12) \\
\hline \multirow{3}{*}{$\begin{array}{r}4500 \mathrm{~Hz} \\
\text { (Bicycle bell) }\end{array}$} & No headphones & \multicolumn{3}{|c|}{0} \\
\hline & Nomal headphone & 0 & $x$ & $x$ \\
\hline & NC headphones & $\mathrm{O}$ & $x$ & $x$ \\
\hline \multirow{3}{*}{$\begin{array}{c}2000 \mathrm{~Hz} \\
\text { (Fire truck) }\end{array}$} & No headphones & \multicolumn{3}{|c|}{$\overline{0}$} \\
\hline & Nomal headphone & 0 & $x$ & $x$ \\
\hline & NC headphones & 0 & $x$ & $x$ \\
\hline \multirow{3}{*}{$\begin{array}{c}200 \mathrm{~Hz} \\
\text { (Car engine) }\end{array}$} & No headphones & \multicolumn{3}{|c|}{$\overline{0}$} \\
\hline & Nomal headphone & 0 & $x$ & $x$ \\
\hline & NC headphones & $x$ & $x$ & $x$ \\
\hline
\end{tabular}

(O) indicating the presence of P300 generation

(x) indicating the absence.

\section{References}

[1] John Polich: Comparison of P300 from Passive and active tasks for auditory and visual stimuli, Int. J, of Psychophysiology, vol.34, (1999) D.F. Pilkey, Happy conservation laws, in: Neural Stresses, J. Frost, ed., Controlled Press, Georgia, 1995, pp. 332-391.

[2] Hiroshi Nittono: Measuring attention to video clips-An application of the prove stimulus technique using event-related brain potentials 【in Japanese】 -Physiology psychology and Mental psychology. vol. 24(1), pp5-18, (2006)

[3] Medical System Educational, Event Related Potential handbook, 【in Japanese】 (2004) pp106-120

[4] Japanese Society of Clinical Neurophysiology: Guideline of measuring event related potential, 【in Japanese】 Journal of Japanese Society of Clinical Neurophysiology vol.25, pp.1115, (1997) 